\title{
Near-Optimum Power Control for Two-Tier SIMO Uplink Under Power and Interference Constraints
}

\author{
Baris Yuksekkaya*, Hazer Inaltekin ${ }^{\dagger}$, Cenk Toker*, and Halim Yanikomeroglu ${ }^{\ddagger}$ \\ ${ }^{*}$ Department of Electrical and Electronics Engineering, Hacettepe University, Ankara, Turkey. \\ Email: \{barisy, cenk.toker\}@ee.hacettepe.edu.tr \\ ${ }^{\dagger}$ Department of Electrical and Electronics Engineering, Antalya International University, Antalya, Turkey. \\ Email: hazeri@antalya.edu.tr \\ $\ddagger$ Department of Systems and Computer Engineering, Carleton University, Ottawa, Ontario, Canada \\ Email: halim@sce.carleton.ca
}

\begin{abstract}
This paper considers optimization of transmit power allocation in a two-tier single input multiple output uplink network. The proposed system model is general enough to cover both emerging heterogeneous network (HetNet) architectures and cognitive radio (CR) networks. The first tier, which is assumed to have one user, represents a macrocell (or a primary user network) and the second tier represents a femtocell (or a secondary user network) in a HetNet (or a CR network). Second tier users share the same resources with the first tier user and they form a single input multiple output multiple access channel to their intended base station while causing interference to the first tier user. Near-optimum allocation of transmission powers maximizing the aggregate communication rate under individual transmission power constraints and a constraint on the total interference power at the first tier user is obtained by considering lowcomplexity signal decoders without interference cancelation at the second tier base station. It is shown that the power allocation is a modified version of the classical water-filling algorithm.
\end{abstract}

\section{INTRODUCTION}

The demand of both society and industry for increasing data rates is currently growing at an unprecedented pace. Emerging next generation multi-tier wireless systems, such as cognitive radio $(\mathrm{CR})$ networks and heterogenous networks (HetNets) with multiple antennas, are among key wireless technologies to meet these needs. The underlying design philosophy in both systems is to make different wireless systems, with different operating conditions, cell sizes and constraints, function in the same frequency block harmoniously. More dynamic and smarter interference-aware resource allocation policies are required for such heterogenous wireless systems to co-exist in the same radio spectrum. In this paper, we focus on the optimization of transmission powers for a two-tier communication network, and obtain the structure of a near-optimum power control policy maximizing aggregate data rates subject to power and interference constraints.

Earlier types of wireless networks are designed to minimize aggregate power consumption in a network while sustaining a target signal to interference plus noise ratio (SINR) [1], which is a well-understood research problem. However, rather than

This work was supported in part by The Scientific and Technological Research Council of Turkey under Grant 2214-A, and in part under Grant $112 \mathrm{E} 024$. attaining target SINR values by using minimum power, the problem for next generation communication networks is to find power control policies that maximize information theoretic bit rates under appropriate system and technological constraints.

Power control problem maximizing communication rates for single tier networks is also relatively well-understood research problem in the literature. For example, Yu et al. focused on maximizing total data rate of a Gaussian vector multiple access channel (MAC) in [2]. They assumed that successive interference cancelation (SIC) can be performed at the receiving base station. Their main result is the generalization of the single user water filling solution to the studied multiuser scenario, where interference is taken as background noise. Inaltekin and Hanly in [3], treated interference as Gaussian noise for a single antenna MAC scenario and showed that the optimum power control policy maximizing the total data rate of users in this case is binary power allocation where users either transmit with full power or do not transmit at all. The main point of difference between our model and those studied in [2] and [3] is that we consider interference constraints to regulate transmission powers in different network tiers.

Next generation wireless systems such as CR networks and HetNets are expected to include multiple tiers of users operating in the same radio spectrum. This leads to a change of design philosophy to distribute transmit power resources among users. To this end, Ghasemi and Sousa focused on an interference power limited power optimization problem for cognitive radio networks consisting of one primary user (PU) and one secondary user (SU) in [4]. In particular, they found the optimum power control policy maximizing the ergodic data rate of the SU while constraining the interference it causes to the PU. Their solution turns out to be a modified water-filling solution where the water level is found using the interference constraint. The single user scenario in [4] is extended to the multiuser scenario in [5] by maximizing the ergodic data rate of a group of users in a secondary network while constraining their average transmit power and the average interference power caused to the primary user. They also showed that the optimal power allocation is a modified version of the waterfilling solution. Our work in this paper extends the results 
reported in [4] and [5] to multi-antenna two-tier networks. In [6], the authors considered the problem of maximizing the data rate of a MIMO multiple access secondary network, while limiting the interference caused to each PU. To simplify the solution, they also limited the total number of SUs in the network and used quantized power levels. They obtained suboptimal solutions that provide some new insights about the structure of the optimum power control. The work in [6] is similar to our work but we do not limit the number of active secondary users and we use continuous power levels rather than quantized ones.

In this paper, we focus on a single input multiple output (SIMO) two-tier network that can be thought of as either representing a SIMO HetNet scenario or a SIMO-CR network scenario. For the HetNet scenario, the first and second tiers respectively correspond to a macrocell and a femtocell. Similarly, for the CR network case, the first tier can be considered as the primary network and the second tier becomes a secondary network. For simplicity, we will assume that there is only one active first tier user (FTU) and multiple second tier users (STU). The use of a single active FTU is a common assumption in the literature, e.g., to maximize total information theoretic capacity by scheduling at any one time only the user with the best channel [7]. The STUs form a multiple access channel with second tier base station (STB). The results that we obtain can be adapted to downlink using uplink-downlink duality [8]. In fact, this is one of our future goals.

The STUs are assumed to have single antenna and the STB is equipped with multiple antennas. Device size limitations force using single antenna at the user side, and multiple antenna users will be considered as a future work. STUs use the same resources as the FTU, and they interfere with the signal reception at the FTU. In our previous work [9], we have assumed that the STB is capable of performing successive interference cancelation (SIC). In this work, we remove this assumption and use the multiple antennas to create a receive filter that mitigates the inter-user interference at the STB. Different from the current work, the paper [9] only considered a single input single output (SISO) communication scenario.

The power optimization problem studied in this paper turns out to be non-convex optimization problem due to interference. Hence, to overcome this difficulty, we utilize interference canceling receive filters to simplify the original problem into a convex one. We show that although the solution for the modified power optimization problem is not necessarily a solution for the original problem, it provides a close lower bound. We further show that there are some nice structural properties of the modified optimization problem that leads to a sum-rate maximizing power allocation in the form of a modified water-filling power allocation solution.

Throughout the paper the scalars are represented by lowercase, vectors are shown as either lowercase bold or uppercase bold overlined, and matrices are shown as uppercase bold characters. The operator $\operatorname{diag}\{\cdot\}$ places the elements on the diagonal of a square matrix, $(\cdot)^{T}$ and $(\cdot)^{H}$ denote the transpose and the Hermitian of a vector/matrix, respectively.
The operator $|\cdot|$ gives the absolute value for scalars.

\section{SYSTEM MOdEL}

We consider a two-tier network, first tier has one user (FTU) with single antenna, and second tier includes $N$ users (STUs) along with their corresponding base station forming a SIMO network. Our model is illustrated schematically in Fig. 1.

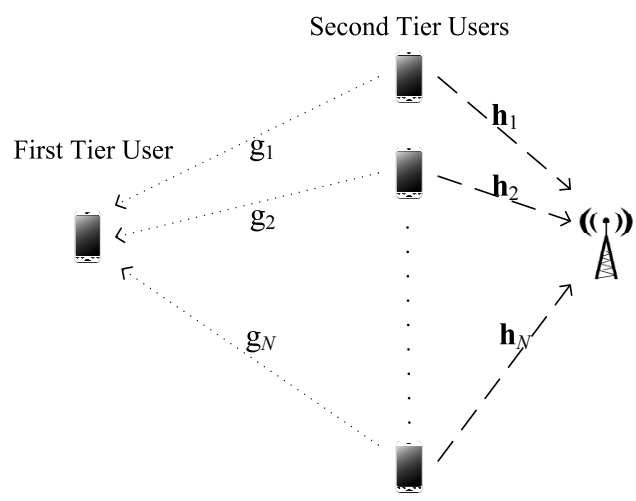

Fig. 1. An illustration of the studied system model with tier-2 users forming a SIMO MAC to their base station and interfering with the tier-1 user.

We focus on the spectrum sharing paradigm [10] for the co-existence of both network tiers in the same radio spectrum, which leads to an interference and noise impaired system. As illustrated in Fig. 1, the STUs form a SIMO-Multiple Access Channel (SIMO-MAC) with second tier base station (STB) and cause interference to the FTU. The vector $\mathbf{h}_{i}$ corresponds to the SIMO channel coefficient vector between the $i^{\text {th }}$ STU and its base station. Similarly, $g_{i}$ corresponds to the interference channel coefficient between the $i^{\text {th }}$ STU and the FTU. It is assumed that direct and interference channel state information, i.e., $\mathbf{h}_{i}$ and $g_{i}$ for $i \in\{1,2, \ldots, N\}$, is perfectly known for all users at the STB, which is a commonly used assumption in the literature [11], [12]. Block-fading model is assumed to model direct and interference channel variations over time. Each STU will transmit with power $P_{i} \in\left[0, P_{\max }\right]$, for $i \in$ $\{1,2, \ldots, N\}$, based on the channel conditions.

Unlike our previous work in [9], we are assuming that successive interference cancellation (SIC) is not available at the STB. However, we added multiple antennas to the STB, and therefore we can still suppress the intra-tier interference by orthogonalizing direct channels from STUs to the STB, as will be explained in Section III of the paper. Unlike [9], these multiple antennas enable user-by-user detection in the receiver. In order to achieve this, the STB should have at least as many antennas as the total number of STUs.

Considering the intra-tier interference at the STB as Gaussian noise, the aggregate communication rate achievable at the STB is given by [13]

$$
R_{\text {total }}(\overline{\mathbf{P}})=\sum_{i=1}^{N} \log _{2}\left(\frac{\operatorname{det}\left[\sigma_{n}^{2} \mathbf{I}+\sum_{i=1}^{N} P_{i} \mathbf{h}_{i} \mathbf{h}_{i}^{H}\right]}{\operatorname{det}\left[\sigma_{n}^{2} \mathbf{I}+\sum_{j \neq i}^{N} P_{j} \mathbf{h}_{j} \mathbf{h}_{j}^{H}\right]}\right),
$$


where $\overline{\mathbf{P}}=\left[\begin{array}{lll}P_{1} & \cdots & P_{N}\end{array}\right]^{T}$, and $\sigma_{n}^{2}$ includes both the variance of the zero mean, additive white Gaussian noise and interference caused by the FTU at the receiver (STB).

The problem is to find the optimal power allocation that maximizes the total data rate of STUs subject to per user power constraints and the total interference power constraint at the FTU, which is formally given as

$$
\begin{array}{lll}
\overline{\mathbf{P}}^{\star}=\underset{\overline{\mathbf{P}}}{\arg \max } & R_{\text {total }}(\overline{\mathbf{P}}) & \\
\text { s.t. } & P_{i} \geq 0, & \forall i, \\
& P_{i} \leq P_{\max }, \quad \forall i, \\
& \sum_{i=1}^{N}\left|g_{i}\right|^{2} P_{i} \leq Q, &
\end{array}
$$

where, the total interference power limit is represented as $Q$.

This is a non-convex power optimization problem due to the intra-tier interference terms in the cost function. To simplify this problem, we first design a receive filter at the STB to diagonalize the direct channels, like the one in [14]. This operation simplifies the power optimization problem in (2) by turning the non-convex objective sum-rate function into a convex function. This means that the solution we obtain after this simplification is the global optimum of the simplified problem but it may not be a global optimum of the original problem due to the data processing inequality [15]. However, to the best of our knowledge, there is no similar work in the literature that combines a MAC without SIC in a multi-tier network that is constrained by the interference caused from one tier to another and this solution is the first step towards finding the optimum power allocation to the original problem.

\section{Receive FILTER Design}

In order to eliminate the intra-tier interference through a receive filter, we first introduce an auxiliary matrix $\hat{\mathbf{H}}_{k}=\left[\begin{array}{llllll}\mathbf{h}_{1} & \cdots & \mathbf{h}_{k-1} & \mathbf{h}_{k+1} & \cdots & \mathbf{h}_{N}\end{array}\right]_{N_{S T B} \times(N-1)}$, i.e., a matrix with all the channel vectors between STUs and STB except for the $k^{\text {th }}$ STU's channel. We then take the singular value decomposition of this matrix as $\hat{\mathbf{H}}_{k}=$

$$
\left[\begin{array}{ll}
\hat{\mathbf{U}}_{k}^{1} & \hat{\mathbf{U}}_{k}^{0}
\end{array}\right]_{N_{S T B} \times N_{S T B}}\left[\begin{array}{c}
\hat{\boldsymbol{\Sigma}}_{k} \\
\mathbf{0}
\end{array}\right]_{N_{S T B} \times(N-1)} \mathbf{V}_{k}^{H} .
$$

Here, $\hat{\boldsymbol{\Sigma}}_{k}$ is an $(N-1) \times(N-1)$ matrix with non-zero singular values of $\hat{\mathbf{H}}_{k}$, and $\hat{\mathbf{U}}_{k}^{0}$ is an $N_{S T B} \times\left(N_{S T B}-(N-1)\right)$ matrix with vectors that are associated with the zero singular values of $\hat{\mathbf{H}}_{k}$. The columns of $\hat{\mathbf{U}}_{k}^{0}$ span the left null space of $\hat{\mathbf{H}}_{k}$, therefore, multiplying $\hat{\mathbf{H}}_{k}$ from the left by the columns of $\hat{\mathbf{U}}_{k}^{0}$ yields zero. Hence, selecting the columns of the receive filter, $\mathbf{U}=\left[\begin{array}{lll}\mathbf{u}_{1} & \cdots & \mathbf{u}_{N}\end{array}\right]$, from the columns of $\hat{\mathbf{U}}_{k}^{0}$, results in an interference free orthogonalized equivalent channel.

Capacity can further be increased by choosing the vector $\mathbf{u}_{k}$ as parallel as possible to $\mathbf{h}_{k}$, i.e., we should choose the vector $\mathbf{u}_{k}$ that maximizes abs $\left(\mathbf{u}_{k}^{H} \mathbf{h}_{k}\right)$. Hence, the receive filter is formed as

$$
\mathbf{U}=\underbrace{\left[\begin{array}{lll}
\mathbf{u}_{1} & \cdots & \mathbf{u}_{N \cdot N_{S T U}}
\end{array}\right]}_{N_{S T B} \times\left(N \cdot N_{S T U}\right)} \longrightarrow \begin{aligned}
& k^{t h} \text { column is a vector } \\
& \text { taken from } \hat{\mathbf{U}}_{k}^{0}, \text { that } \\
& \text { maximizes abs }\left(\mathbf{u}_{k}^{H} \mathbf{h}_{k}\right) .
\end{aligned}
$$

then the effective channel after the receive filter becomes $\mathbf{H}_{e f f}=\mathbf{U}^{H} \mathbf{H}=\operatorname{diag}\left\{\mu_{1}, \ldots, \mu_{N}\right\}$.

We observe that the effective channel $\mathbf{H}_{e f f}$ is a diagonal matrix, i.e., each STU communicates in a point to point scenario where $i^{\text {th }}$ STU has a channel coefficient $\mu_{i}$; in other words, the receive filter eliminates the intra-tier interference. We also observe that the sum rate is just the sum of data rates achieved by each user. This sum-rate can be shown as

$$
R^{\prime}(\overline{\mathbf{P}})=\sum_{i=1}^{N} \log _{2}\left(1+\frac{P_{i}\left|\mu_{i}\right|^{2}}{\sigma_{n}^{2}}\right) .
$$

where $R^{\prime}$ is the aggregate communication rate after the use of the receive filter.

The important point here is the fact that the designed receive filter may not be unitary, i.e., $\mathbf{U}^{H} \mathbf{U} \neq \mathbf{I}$. Hence, the noise at the receive filter output at the STB may turn out to be colored Gaussian noise. It is known that in general colored noise gives a rate $C_{\text {colored }} \leq C_{\text {white }}$ since we assume that noise is spatially white in the decoding process [16]. Therefore, we show the resulting sum-rate as $R^{\prime}(\overline{\mathbf{P}})$ in (4), and this sum-rate is lower than or equal to the rate in (1), i.e., $R^{\prime}(\overline{\mathbf{P}}) \leq R_{\text {total }}(\overline{\mathbf{P}})$. The equality, hence optimality, occurs when $\mathbf{U}^{H} \mathbf{U}=\mathbf{I}$.

\section{Near-Optimum Power Allocation}

After the diagonalization of the channel by the receive filter $\mathbf{U}$, user-by-user detection is possible. The total data rate becomes the sum of each STU's individual data rate, then the new optimization problem can be written as

$$
\begin{array}{cll}
\overline{\mathbf{P}}^{\star}=\underset{\overline{\mathbf{P}}}{\arg \max } & \sum_{i=1}^{N} \log _{2}\left(1+P_{i}\left|\mu_{i}\right|^{2}\right) & \\
\text { s.t. } & P_{i} \geq 0, & \forall i, \\
& P_{i} \leq P_{\max }, & \forall i, \\
& \sum_{i=1}^{N}\left|g_{i}\right|^{2} P_{i} \leq Q, &
\end{array}
$$

where, without loss of generality, the variance of the Gaussian noise is taken as 1 .

It is obvious that the cost function of this problem is different than that of our previous work in [9], therefore, the allocation procedure is different too. The optimum power allocation for this problem is given in the theorem below,

Theorem 1: For a two-tier network, that contains one FTU and multiple STUs where the STB is using a receive filter to remove the inter-user interference, the optimum power allocation under power constraints and an interference constraint on the FTU is given by the modified water-filling power allocation algorithm. The solution to this problem is

$$
\begin{gathered}
P_{i}^{\star}=\min \left[\left(\frac{1}{\ln (2) \lambda\left|g_{i}\right|^{2}}-\frac{1}{\left|\mu_{i}\right|^{2}}\right), P_{\max }\right]^{+}, \\
\sum_{i=1}^{N} \min \left[\left(\frac{1}{\ln (2) \lambda\left|g_{i}\right|^{2}}-\frac{1}{\left|\mu_{i}\right|^{2}}\right), P_{\max }\right]^{+}\left|g_{i}\right|^{2}=Q,
\end{gathered}
$$


Proof: To solve the problem in (5), we need to find the Lagrangian,

$$
L=-\sum_{i=1}^{N} \log _{2}\left(1+P_{i}\left|\mu_{i}\right|^{2}\right)+\lambda\left(\sum_{i=1}^{N}\left|g_{i}\right|^{2} P_{i}-Q\right)
$$

and equate its first derivative to zero [17], i.e.,

$$
\left.\frac{\partial L}{\partial P_{i}}\right|_{P_{i}=P_{i}^{\star}}=\frac{1}{\ln (2)} \frac{\left|\mu_{i}\right|^{2}}{1+P_{i}^{\star}\left|\mu_{i}\right|^{2}}-\lambda\left|g_{i}\right|^{2}=0 .
$$

We incorporate the first and second constraints to the solution at the next step as follows

$$
P_{i}^{\star}=\min \left[\left(\frac{1}{\ln (2) \lambda\left|g_{i}\right|^{2}}-\frac{1}{\left|\mu_{i}\right|^{2}}\right), P_{\max }\right]^{+} \text {. }
$$

Equation (9) defines the modified water-filling power allocation with $\frac{1}{\ln (2) \lambda\left|g_{i}\right|^{2}}$ as the water level. This level is found by solving $\sum_{i=1}^{N}\left|g_{i}\right|^{2} P_{i}^{\star}=Q$ for the Lagrange multiplier $\lambda$.

The optimum power allocation solution in (6), first the water level is defined such that the interference constraint is not violated. If there are users that need more power than that level then they are set to have zero power, and if the power of an STU is higher than $P_{\max }$ then that user is set to work with $P_{\max }$. Next a set is created which includes STUs with neither zero power nor $P_{\max },\left(S=\left\{i: 0<P_{i}<P_{\max }\right\}\right)$. Afterwords the remaining power is calculated using the interference constraint and it is distributed evenly within the users in $S$. Again, if any user is allocated with power more than $P_{\max }$, that user's power is set to $P_{\max }$ and it is discarded from the set $S$. This power distribution continues until interference constraint is satisfied.

As follows, the solution is similar to the conventional waterfilling solution with a few structural differences. The first and probably the most clear difference is that the process is actually interference-filling rather than a power-filling process because the water level is found using the total interference constraint in (7). Moreover, as another difference, the water level, in (6), has an additional multiplier (interference channel power gain $\left|g_{i}\right|^{2}$ ) in its denominator. Furthermore, note that transmit power of each antenna is upper limited rather than a per user power limit. These differences are the reasons to call the solution modified water-filling solution. In addition, one has to remember that this solution is optimum for the simplified problem given in (5), but it may not be a global optimum solution of the original problem in (2). Therefore we call this solution near-optimum.

It can be obviously seen that if the interference channel power gain $\left(\left|g_{i}\right|^{2}\right)$ increases, then the allocated power to the related user decreases, i.e., the interference channel power gain effects each user's water level directly. Therefore, increasing the interference channel power gain, decreases the related user data rate and eventually decreases the total data rate.

\section{Simulations And Results}

In the simulations the model has 1 FTU and varying number of STUs $(3,5,7,9)$ each with single antenna. The number of antennas in the STB is equal to the total number of STUs (i.e., $3,5,7,9)$. In all of the simulations, maximum power per user is set to 1 Watt, interference constraint $Q$ is chosen as 1 Watt, and noise is zero-mean circularly symmetric additive white Gaussian noise with variance $\sigma^{2}$. Without loss of generality, we will assume the noise variance as $\sigma^{2}=1$ in the simulations. Mean of the power gains of the channels between STUs and STB is constant at unity but the mean channel power gains between the FTU and STUs are varying. For each channel configuration, we plot the ergodic sum-rates achieved by averaging over long time intervals. That is, for any given $\mathbf{H}=\left[\begin{array}{llll}\mathbf{h}_{1} & \mathbf{h}_{2} & \cdots & \mathbf{h}_{N}\end{array}\right]$ and $\mathbf{g}=\left[\begin{array}{llll}g_{1} & g_{2} & \cdots & g_{N}\end{array}\right]^{T}$, we first solve the optimum power control problem posed in (5). Then, we average data rates over $\mathbf{H}$ and $\mathbf{g}$ to obtain ergodic sum-rates $\mathrm{E}_{\mathbf{H , g}, \mathbf{g}}\left[R\left(\boldsymbol{P}^{\star}(\mathbf{H}, \mathbf{g})\right)\right]$. These rates are plotted as a function of the number of STUs, and the effect of different fading parameters (means of channel power gains) are investigated.

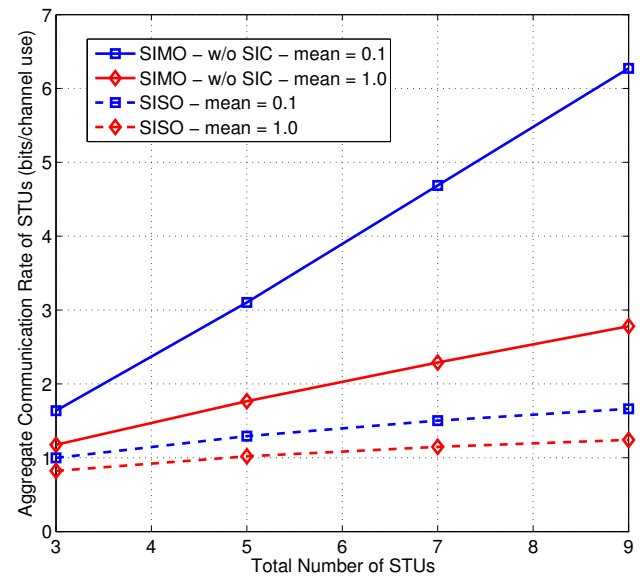

Fig. 2. Comparison of SISO scenario with SIC versus SIMO scenario without SIC.

In Fig. 2, both STU-STB and STU-FTU channels are chosen as Rayleigh fading channels. Sum-rate is observed for both SISO scenario with SIC and SIMO scenario without SIC for different number of STUs and different interference channel means. As expected, an increase in the mean of the power gains of interference channels results in a decrease in the sum-rate. This result can be seen in the power allocation in (6). As seen, increasing $\left|g_{i}\right|^{2}$, decreases $\hat{P}_{i}$, that is, the power of the related antenna decreases; this, in turn, decreases the data rate of the corresponding user. Moreover, it can be seen that the multiple antennas in the SIMO scenario do not only eliminate the interference, but they also increase the data rate of STUs. In the SIMO scenario increasing the means of interference channel power gains decreases the data rate more in comparison to the decrease in the SISO scenario. 


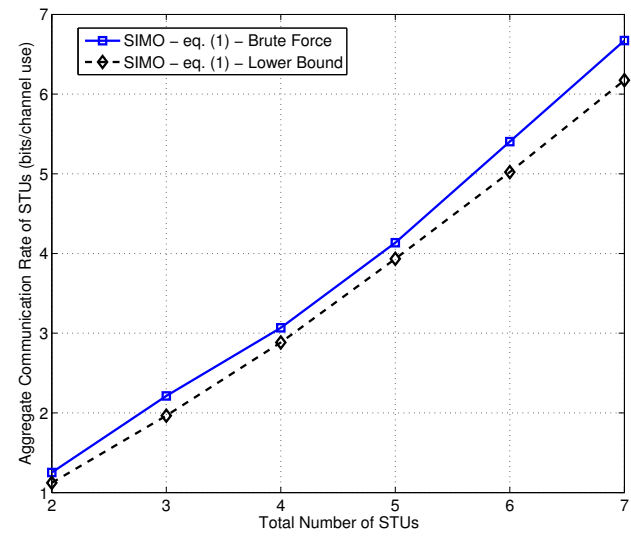

Fig. 3. Comparison of SISO scenario with SIC versus SIMO scenario without SIC.

As mentioned earlier, the allocation found by the problem in (5) may not give a global optimum solution to the original problem in (2). Actually, using the $P_{i}^{\star}$ 's found by the modified water-filling algorithm in the rate formula (6) achieves a lower bound for the optimum aggregate communication rate. To find out how close this lower bound is, we need to find a brute force maximum of the data rate in (1) by using an exhaustive search method and compare the results.

In Fig. 3, the lower bound and the exhaustive search results of the optimum rate for the problem in (2) is compared. It can be observed that the brute force solution and the lower bound are close to each other. This suggests that the lower bound, found using the solution of the simplified problem, is close to the optimum solution of the original problem.

\section{CONCLUSIONS}

Data rate maximization for a two-tier network framework that covers both a HetNet and a CR network is examined in this paper. First tier may represent a macrocell of a HetNet or a primary network in cognitive radio network, and the second tier can be thought of as a femtocell or a secondary network for a Hetnet, or a cognitive radio network, respectively. It is assumed that all users share the same resources, hence, in the uplink the STUs cause interference to the FTU. The problem is to maximize the aggregate data rate of STUs while constraining the total interference caused to the FTU.

It is assumed that the STB is not capable of doing successive interference cancelation (SIC), hence, suffers from inter-user interference. This causes the cost function in (2) to be nonconvex. In order to mitigate this interference and have a simplified and convex cost function, a receive filter is designed to orthogonalize each channel of each antenna.

In the optimization problem, along with individual power constraints, the interference caused by STUs to the FTU is held under a threshold level. The optimum power allocation, for the simplified problem, is found as a modified water-filling power allocation where the power level is found using the total interference power constraint. To the best of our knowledge, this solution can be thought as a generalized version to the similar solutions in the literature. Also, this solution is a close lower bound and a near-optimum solution to the original nonconvex problem. Simulations for Rayleigh fading scenarios showed the evolution of the total data rate with respect to number of STUs and varying channel parameters. It is seen that the interference channel, i.e., the channel between STUs and FTU, directly effects the data rate of users and increasing the number of antennas not only helps eliminating the interference but also helps increasing the data rate.

Using the optimum solution of the simplified problem in the original communication rate in (1), we found a lower bound to the original problem in (2). To show that this lower bound is a tight bound, we compared it with a brute force solution of the original problem.

\section{REFERENCES}

[1] S. V. Hanly and D. N. C. Tse, "Power control and capacity of spread spectrum wireless networks," Automatica, vol. 35, no. 12, pp. 19872012, Dec. 1999.

[2] W. Yu, W. Rhee, S. Boyd, and J. M. Cioffi, "Iterative water-filling for Gaussian vector multiple-access channels," IEEE Trans. Inf. Theory, vol. 50, no. 1, pp. 145-152, 2004

[3] H. Inaltekin and S. V. Hanly, "Optimality of binary power control for the single cell uplink," IEEE Trans. Inf. Theory, vol. 58, no. 10, pp. 6484-6498, Oct 2012.

[4] A. Ghasemi and E. S. Sousa, "Fundamental limits of spectrum-sharing in fading environments," IEEE Trans. Wireless Commun., vol. 6, no. 2, pp. 649-658, Feb. 2007.

[5] E. Nekouei, H. Inaltekin, and S. Dey, "Throughput scaling in cognitive multiple access with average power and interference constraints," IEEE Trans. Signal Process., vol. 60, no. 2, pp. 927-946, 2012.

[6] M. Naeem, U. Pareek, and D. C. Lee, "Interference-aware joint user selection and quantised power control schemes for uplink cognitive multiple-input multiple-output system," IET Commun., vol. 5, no. 16, pp. 2266-2274, 2011.

[7] P. Viswanath, D. N. C. Tse, and R. Laroia, "Opportunistic beamforming using dumb antennas," IEEE Trans. Inf. Theory, vol. 48, no. 6, pp. 1277$1294,2002$.

[8] P. Viswanath and D. N. C. Tse, "Sum capacity of the vector Gaussian broadcast channel and uplink-downlink duality," IEEE Trans. Inf. Theory, vol. 49, no. 8, pp. 1912-1921, 2003.

[9] B. Yuksekkaya, H. Inaltekin, and C. Toker, "Optimum uplink power control under power and interference constraints," in IEEE 78th Vehicular Technol. Conf. (VTC Fall-2013), 2013, pp. 1-5.

[10] A. Goldsmith, S. A. Jafar, I. Maric, and S. Srinivasa, "Breaking spectrum gridlock with cognitive radio: An information theoretic perspective," Proc. IEEE, vol. 97, no. 5, pp. 894-914, May 2009.

[11] L. Li and A. J. Goldsmith, "Capacity and optimal resource allocation for fading broadcast channels. I. Ergodic capacity," IEEE Trans. Inf. Theory, vol. 47, no. 3, pp. 1083-1102, 2001.

[12] I. C. Wong and B. L. Evans, "Optimal downlink OFDMA resource allocation with linear complexity to maximize ergodic rates," IEEE Trans. Wireless Commun., vol. 7, no. 3, pp. 962-971, 2008.

[13] M. Chiani, M. Z. Win, and H. Shin, "Capacity of MIMO systems in the presence of interference," in IEEE Global Telecommunications Conference, 2006, pp. 1-6.

[14] D. Gesbert, M. Kountouris, R. W. Heath, C. Chae, and T. Salzer, "Shifting the MIMO paradigm," IEEE Signal Process. Mag., vol. 24, no. 5, pp. 36-46, 2007.

[15] T. M. Cover and J. A. Thomas, Elements of Information Theory, John Wiley \& Sons, 2012

[16] R. W. Heath, M. Airy, and A. J. Paulraj, "Multiuser diversity for MIMO wireless systems with linear receivers," in IEEE Thirty-Fifth Asilomar Conf. on Sig., Sys. and Comp., 2001, vol. 2, pp. 1194-1199.

[17] S. Boyd and L. Vandenberghe, Convex Optimization, Cambridge University Press, New York, NY, USA, 2004. 